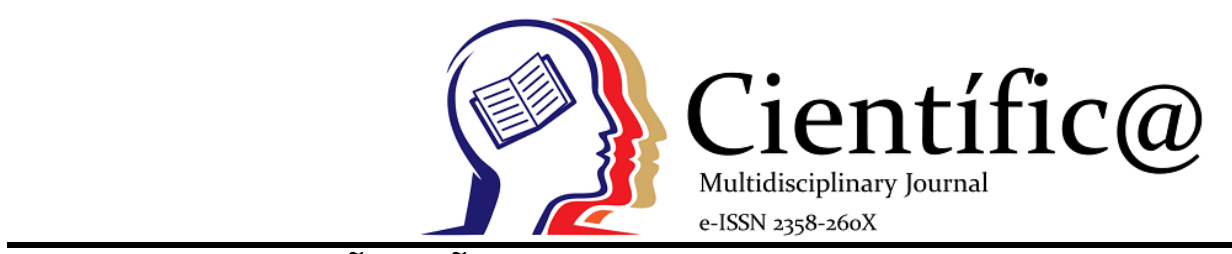

\title{
AVALIAÇÃO NÃO LINEAR DOS ESFORÇOS INTERNOS EM CONÓIDES CILÍNDRICOS
}

\section{EVALUATION OF NON LINEAR EFFORTS IN INTERNAL CYLINDRICAL CONOIDS}

\author{
Danielly Luz Araújo de Morais ${ }^{1}$
}

\begin{abstract}
${ }^{1}$ Autor para correspondência; Mestre em Engenheira civil - Faculdade Evangélica de Goianésia. daniellyluzaraujo@hotmail.com
\end{abstract}

Info

Recebido: 08/2019

Publicado: 04/2020

DOI: $10.29247 / 2358-260 X .2020 v 7 i 1.3919$

ISSN: 2358-260X

Palavras-Chave
Análise não linear, Casca conoidal
cilíndrica, Esforços solicitantes, Método dos
elementos finitos.
Keywords:
Nonlinear analysis, Cylindrical conoidal
shell, Requesting effort, Finite element
method.

\begin{abstract}
Resumo
Em arquiteturas da atualidade, percebe-se uma abordagem moderna demandando tendências de soluções estruturais complexas no design estrutural e o conóide é uma forma geométrica simples que confere requisitos de beleza e sofisticação a diversas edificações deste contexto. A obtenção dos esforços solicitantes para o dimensionamento de uma estrutura em concreto com esta forma geométrica não é tão simples e exige conhecimentos avançados. Objetivando-se a avaliação do caminho não linear dos esforços de membrana e de momentos, este trabalho apresenta uma investigação numérica estática não linear, via método dos elementos finitos (MEF), de cascas conoidais de geometria cilíndrica, submetida ao carregamento transversal uniformemente distribuído aplicado incrementalmente ao longo da sua superfície. Com este trabalho ilustra-se a importância da análise não linear ao identificar como as posições dos esforços máximos, cruciais para o dimensionamento estrutural, modificam-se ao longo da
\end{abstract} incrementação de carga. Comparando-se os esforços de flexão de duas geometrias diferentes observa-se a influência de diferentes modos de vibração na trajetória da topologia, com prevalência de momentos fletores positivos no centro, negativos nas extremidades e, com relação aos esforços de torção, sua concentração máxima prevalece perto das bordas curvas. Observam-se no início da trajetória do conóide de duas bordas curvas que as máximas regiões comprimidas não estão próximas às bordas retas, como no conóide de apenas uma borda curva. Além disso, é importante destacar a ocorrência de picos de esforços cisalhantes nas bordas curvas, pois nas demais localidades da casca os esforços cisalhantes tem menor intensidade.

\footnotetext{
Abstract

In present architectures, a modern approach to architectures is perceived demanding trends of complex structural solutions design and the conoid is a simple geometric form that gives beauty and sophistication requirements to various buildings in this context. Obtaining the requisite efforts for sizing a concrete structure with this geometric shape is not so simple and requires advanced knowledge. Aiming to evaluate the nonlinear path of the moments and membrane stresses, this work presents a nonlinear static numerical investigation, via the finite element method (MEF), of cylindrical conoidal shells, subjected to uniformly distributed transverse loading incrementally applied along of its surface. This work illustrates the importance of nonlinear analysis to identify how the maximum stress positions, crucial for structural design, change along the load increment. Comparing the bending forces of two different geometries, one can observe the influence of different modes of vibration on the path of the topology, with prevalence of positive bending moments in the center, negative in the extremities and, in relation to the torsional efforts, its maximum concentration prevails near the Curved edges. It is observed at the beginning of the two curved edge conoid trajectory that the maximum compressed regions are not close to the straight edges, as in the single curved edge conoid. In addition, it is important to highlight the occurrence of shear stress peaks at the curved edges, since in the other locations of the shell the shear stress is less intense.
} 


\section{INTRODUÇÃO}

A utilização de geometrias curvas na cobertura de edificações revolucionou a arquitetura e ganhou espaço nos últimos tempos. Além da busca pelo desenho inovador das fachadas, nem sempre é possível a utilização de formas convencionais em estruturas que necessitem grandes vãos. Chakravorty e Das (2009) especificam que a casca conoidal tem grande aplicação

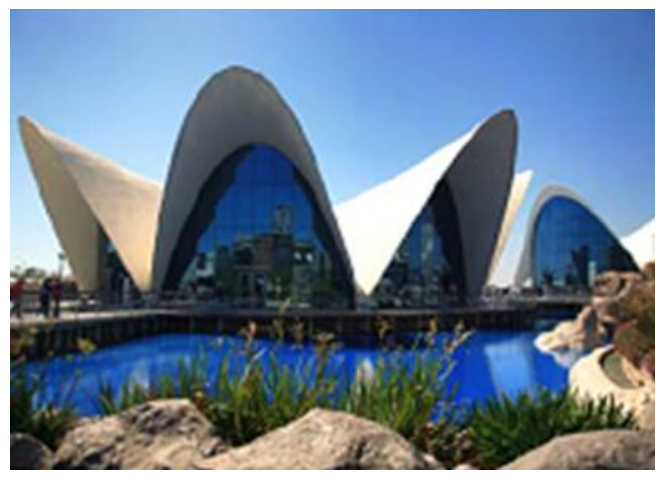

(a) para coberturas que necessitam de grandes vãos. Como opção para aferir arrojo e boa resistência mecânica às coberturas de suas obras, arquitetos renomados como Félix Candela e Oscar Niemeyer utilizaram a forma da casca conoidal. Dentre várias de suas obras destacamse o Bacardi Rum Factory, no México, e a Fundação Getúlio Vargas, em São Paulo, ilustrados na figura 1.

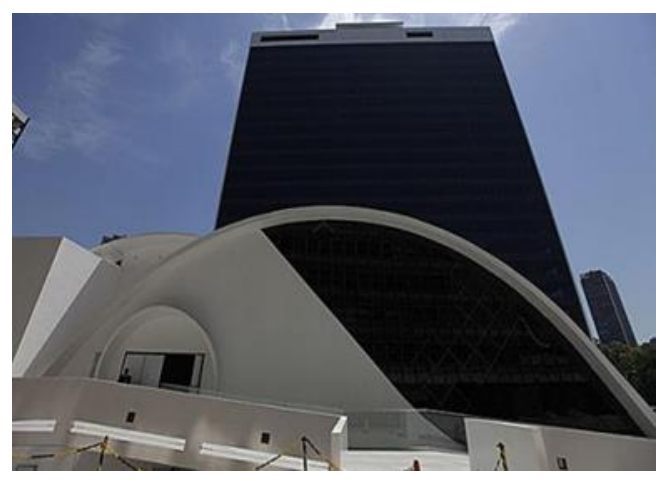

(b)

Figura 1. Cascas conoidais (a) Bacardi Rum Factory: Félix Candela (Fonte: STRUCTURAE, 2016) (b) Fundação Getúlio Vargas: Oscar Niemeyer (Fonte: FGV, 2016)

O conóide é um tipo de casca de translação formada por uma curva, contida num plano, transladada sobre outra curva, ou reta, contida em outro plano perpendicular ao primeiro. Apesar de haver estudos que versam sobre análises de cascas conoidais por métodos numéricos (Bandyopadhyay et all (1995); Das e Chakravorty (2009); Sahoo (2013); Cavalcanti (2015); Gonçalves e Soares (2016)) e também analíticos (Ghosh e Bandyopadhyay (1990); Stavridis (1998); Debongnie (1978)), o seu comportamento não-linear ainda não está totalmente compreendido.

Objetiva-se com este trabalho avaliar o comportamento estático não-linear dos esforços solicitantes de uma casca conoidal cilíndrica de projeção retangular no plano horizontal, bordas curvas cilíndricas e restrição às translações em todas as bordas via MEF utilizando-se o programa comercial Abaqus 6.11 ®. As não linearidades do problema são advindas da geometria que adicionam termos não lineares às equações de equilíbrio influenciando no comportamento da casca conoidal, como pode ser observado na formulação matemática apresentada a seguir. Para esse estudo, efetua-se uma análise paramétrica variando-se as alturas das bordas curvas dos conóides e avaliam-se as trajetórias não lineares dos esforços internos durante a incrementação de carga distribuída uniformemente ao longo da superfície.

\section{FORMULAÇÃO MATEMÁTICA}

Uma importante fase de avaliação de um problema físico consiste na formulação do problema matemático, pois isto rege a análise. A modelagem geométrica proposta em Morais (2017) para a casca conoidal está ilustrada na figura 2, visualiza-se que o conóide está sujeito a um carregamento uniformemente distribuído $\bar{q}$ ao longo de sua superfície. Além disso, a 
modelagem numérica considera material elástico-linear, homogêneo e isotrópico de módulo de elasticidade $E$, coeficiente de Poisson e densidade.

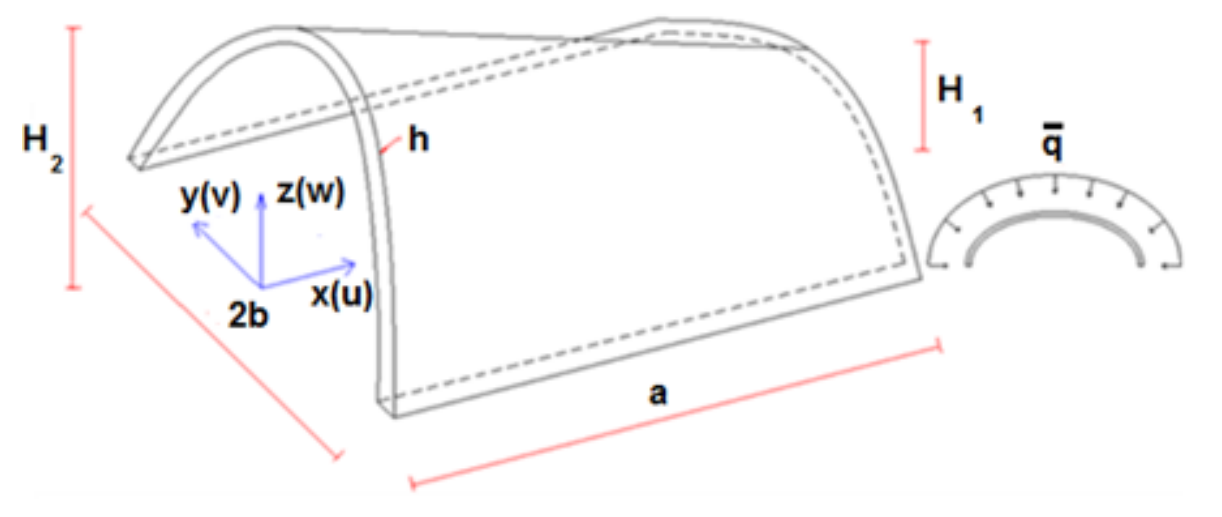

Figura 2. Casca conoidal

A geometria é definida a partir da espessura $h$, do comprimento no plano $a$, da largura no plano $2 b$ e das alturas máximas das bordas curvas cilíndricas, $H_{2}$ e $H_{1}$. Segundo Debongnie (1978), pela teoria de Marguerre a curvatura da superfície da casca é considerada no sistema de coordenadas cartesianas, não envolvendo raios de curvatura, os campos de deslocamentos $u, v$ e $w$ referem-se às direções $x, y$ e $z$, respectivamente.

Para descrever a superfície da casca conoidal de variação das bordas curvas cilíndricas é apresentada por Morais (2017) a seguinte equação:

$$
\bar{z}(x, y)=-\left(H_{2}+\frac{\left(H_{1}-H_{2}\right)}{a} x\right) \sqrt{1-\frac{y^{2}}{b^{2}}}
$$

Bandyopadhyay e Ghosh (1990) representam a carga vertical $(\bar{q})$ distribuída por unidade de área da superfície da casca, sendo $q$ a magnitude da carga distribuída na superfície da casca:

$$
\bar{q}=q\left[1+\left(\frac{\partial \bar{z}(x, y)}{\partial x}\right)^{2}+\left(\frac{\partial \bar{z}(x, y)}{\partial y}\right)^{2}\right]^{1 / 2}
$$

Como hipótese básica, considera-se que a casca conoidal seja uma placa com uma imperfeição inicial na forma da casca conoidal. Sendo assim, as hipóteses de Kirchhoff-Love para placas esbeltas (Almroth e Brush, 1975) é utilizada na formulação matemática do conóide e os campos de deslocamentos são representados pela equação (3).

$$
\bar{u}=u+z \beta_{x} \quad \bar{v}=v+z \beta_{y} \quad \bar{w}=w+\bar{z}(x, y)
$$

Os campos de deformações não lineares no plano médio da casca conoidal abatida e suas mudanças de curvatura são dados pela equação (4), considerandose a teoria de Von Kárman.

$\varepsilon_{x}=u_{, x}+\frac{1}{2}\left(w_{, x}{ }^{2}+2 w_{, x} \bar{z}(x, y)_{, x}\right)$

$$
\varepsilon_{y}=v_{, y}+\frac{1}{2}\left(w_{, y}{ }^{2}+2 w_{, y} \bar{z}(x, y)_{, y}\right)
$$

$$
\gamma_{x y}=u_{, y}+v_{, x}+w_{, x} w_{, y}+w_{, x} \bar{z}(x, y)_{, y}+w_{, y} \bar{z}(x, y)_{, x}
$$

$$
\kappa_{x}=-w^{, x x} \quad \kappa_{y}=-w_{, y y} \quad \kappa_{x y}={ }^{,}, x y
$$

As equações (5) fornecem as deformações em qualquer ponto da casca a partir das deformações no plano médio e das mudanças de curvatura. 
$\bar{\varepsilon}_{x}=\varepsilon_{x}+z \kappa_{x} \quad \bar{\varepsilon}_{y}=\varepsilon_{y}+z \kappa_{y} \quad \bar{\gamma}_{x y}=\gamma_{x y}+2 z \kappa_{x y}$

As relações constitutivas tensão-deformação para o material elástico, linear, homogêneo e isotrópico em qualquer ponto ao longo da espessura da casca são dadas por:

$$
\begin{gathered}
\bar{\sigma}_{x}=\frac{E}{1-v^{2}}\left(\bar{\varepsilon}_{x}+v \bar{\varepsilon}_{y}\right) \quad \bar{\sigma}_{y}=\frac{E}{1-v^{2}}\left(\bar{\varepsilon}_{y}+v \bar{\varepsilon}_{x}\right) \\
\bar{\tau}_{x y}=\frac{E}{2(1+v)} \bar{\gamma}_{x y}
\end{gathered}
$$

As relações constitutivas para os esforços normais e cisalhantes no plano são obtidas pela integração das tensões ao longo da espessura do conóide, equações (7), assim como para os momentos internos, equações (8).

$$
\begin{aligned}
& N_{x}=\int_{-\frac{h}{2}}^{\frac{h}{2}} \bar{\sigma}_{x} d z \quad N_{y}=\int_{-\frac{h}{2}}^{\frac{h}{2}} \bar{\sigma}_{y} d z \quad N_{x y}=\int_{-\frac{h}{2}}^{\frac{h}{2}} \bar{\tau}_{x y} d z \\
& \mathrm{C}\left[\left\{\left(u_{, x}+\frac{1}{2}\left(w_{, x}{ }^{2}+2 w_{, x} \bar{z}(x, y)_{, x}\right)\right)+v\left(v_{, y}+\frac{1}{2}\left(w_{, y}{ }^{2}+2 w_{, y} \bar{z}(x, y)_{, y}\right)\right)\right\}_{, x}\right. \\
& \left.+\frac{1-v}{2}\left\{\left(u_{, y}+v_{, x}+w_{, x} w_{, y}+w_{, x} \bar{z}(x, y)_{, y}+w_{, y} \bar{z}(x, y)_{, x}\right)\right\}_{, y}\right]=0 \\
& \mathrm{C}\left[\frac{1-v}{2}\left\{\left(u_{, y}+v_{, x}+w_{, x} w_{, y}+w_{, x} \bar{z}(x, y)_{, y}+w_{, y} \bar{z}(x, y)_{, x}\right)\right\}_{, x}\right. \\
& \left.+\left\{\left(v_{, y}+\frac{1}{2}\left(w_{, y}{ }^{2}+2 w_{, y} \bar{z}(x, y)_{, y}\right)\right)+v\left(u_{, x}+\frac{1}{2}\left(w_{, x}{ }^{2}+2 w_{, x} \bar{z}(x, y)_{, x}\right)\right)\right\}_{, y}\right]=0 \\
& -D\left[\left\{w_{, x x}+v w_{, y y}\right\}_{, x x}+2(1-v)\left(\frac{1}{2}\left(w_{, x y}+w_{, y x}\right)\right)_{, x y}+\left(w_{, y y}+v w_{, x x}\right)_{, y y}\right] \\
& -\mathrm{C}\left[\left(\left(u_{, x}+\frac{1}{2}\left(w_{, x}{ }^{2}+2 w_{, x} \bar{z}(x, y)_{, x}\right)\right)+v\left(v_{, y}+\frac{1}{2}\left(w_{, y}{ }^{2}+2 w_{, y} \bar{z}(x, y)_{, y}\right)\right)\right) w_{, \mathrm{x}}\right. \\
& \left.+\frac{1-v}{2}\left(u_{, y}+v_{, x}+w_{, x} w_{, y}+w_{, x} \bar{z}(x, y)_{, y}+w_{, y} \bar{z}(x, y)_{, x}\right) w_{, \mathrm{y}}\right]_{, \mathrm{x}} \\
& -\mathrm{C}\left[\left(\left(v_{, y}+\frac{1}{2}\left(w_{, y}{ }^{2}+2 w_{, y} \bar{z}(x, y)_{, y}\right)\right)+v\left(u_{, x}+\frac{1}{2}\left(w_{, x}{ }^{2}+2 w_{, x} \bar{z}(x, y)_{, x}\right)\right)\right) w_{, \mathrm{y}}\right. \\
& \left.+\frac{1-v}{2}\left(u_{, y}+v_{, x}+w_{, x} w_{, y}+w_{, x} \bar{z}(x, y)_{, y}+w_{, y} \bar{z}(x, y)_{, x}\right) w_{, \mathrm{x}}\right]_{, \mathrm{y}}=\bar{q}
\end{aligned}
$$

$$
M_{y}=\int_{-\frac{h}{2}}^{\frac{h}{2}} z \bar{\sigma}_{y} d z
$$

As equações não lineares de equilíbrio são obtidas partindo-se do critério da energia potencial estacionária e através das ferramentas do cálculo variacional são obtidas três equações diferenciais não lineares de equilíbrio, equações (9)-(11). Verifica-se a presença dos deslocamentos $u, v$ e $w$ sugerindo o acoplamento de todos os campos de deslocamentos, além de não linearidades cúbicas e quadráticas e do tipo variação superficial influenciando no comportamento estrutural do conóide. 
Onde $C=E h /\left(1-\square^{2}\right)$ e $D=E h^{3} / 12\left(1-\square^{2}\right)$ são, respectivamente, os parâmetros de rigidez de membrana e de flexão.

Ainda segundo Morais (2017), objetivar uma análise analítica da casca conoidal exige uma solução muito complicada, ou até mesmo impossível, para as equações de equilíbrio e o MEF é um método numérico para obtenção de aproximações aos resultados destes problemas. Nesse sentido, as análises estáticas não lineares que serão apresentadas no próximo capítulo se darão somente a partir dos resultados obtidos por modelagem computacional via MEF utilizando o programa comercial $A B A Q U S$ 6.11®.

Alguns aspectos da formulação dos elementos finitos devem ser destacados, pois influenciam no comportamento do problema apresentado. Dentre estes, os graus de liberdade são as variáveis fundamentais de análise e os de translação e rotação são os mais corriqueiros em problemas de engenharia. Os nós também são itens importantes destacar, estes são os pontos onde são aplicadas as condições de contorno, carregamentos e são calculados os graus de liberdade dos elementos e nos demais pontos, as variáveis são obtidas por interpolação a partir das informações obtidas no nó.

O elemento utilizado é o STRI3, ilustrado na Figura 3. Segundo o manual do ABAQUS 6.11®, a formulação satisfaz a teoria de Kirchhoff, como na formulação apresentada neste capítulo, e considera somente pequenas deformações. Para o STRI3, não há alterações nas análises com relação à mudança da espessura do elemento durante a deformação, é um elemento de casca, triangular, contendo três nós sem existência de nós intermediários, resultando em interpolação linear, ou de primeira ordem. $\mathrm{O} A B A Q U S$ 6.11 R aplica técnicas numéricas para integrar ao longo do volume de cada elemento usando quadratura Gaussiana para a maioria dos casos e avaliando a resposta do material em cada ponto de integração, ou pontos de Gauss, ilustrados pelo símbolo "X” na Figura 3.

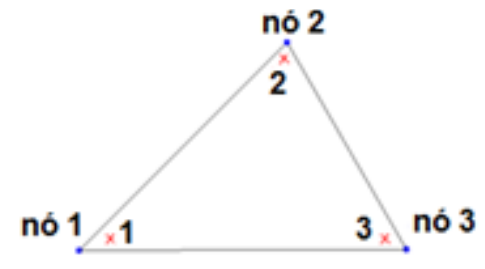

Figura 3. Elemento finito STRI3 (Morais, 2017)

As não linearidades do problema são devidas à geometria e são descritas nos termos não lineares nas equações de equilíbrio e, para solucioná-las o programa Abaqus ${ }^{\circledR}$ combina processos de iteração e de incrementação do carregamento aplicado através do método de Newton-Raphson. Quando na trajetória de equilíbrio há perda da capacidade de carga utiliza-se o método numérico de Riks, método de procedimento de comprimento de arco no qual tanto os graus de liberdade a cada passo de aplicação do carregamento, quanto à magnitude de carregamento imposta à estrutura funcionam como incógnitas a resolver.

\section{RESULTADOS NUMÉRICOS}

Considera-se uma casca conoidal constituída de material elástico, linear, homogêneo e isotrópico com módulo de elasticidade $\mathrm{E}=14 \mathrm{GPa}$, coeficiente de Poisson $\square \square \square \square \square$ e densidade $\square=2548,42 \mathrm{~kg} / \mathrm{m}^{3}$ submetida a um carregamento uniformemente distribuído na superfície, $\bar{q}$, e deslocamentos translacionais impedidos nas quatro bordas. A geometria é a apresentada na figura 2, de comprimento $\mathrm{a}=6 \mathrm{~m}$, largura $2 \mathrm{~b}=8 \mathrm{~m}$ e espessura $\mathrm{h}=0,06 \mathrm{~m}$. As duas bordas no sentido de "a" são consideradas retas, a altura da borda curva $\mathrm{H}_{2}=1,50 \mathrm{~m}$, localizada na borda $\mathrm{x}=0$, e a borda $\mathrm{H}_{1}$, localizada na borda $\mathrm{x}=\mathrm{a}$, é considerada em duas variações: $H_{1}=0 \mathrm{~m}$ para a Geometria 1 e $H_{1}=1,50 \mathrm{~m}$ para a Geometria 2. A 
malha de 9600 elementos finitos STRI3 utilizada no

programa Abaqus ${ }^{\circledR}$ é ilustradas na figura 4 para a

Geometria 1.

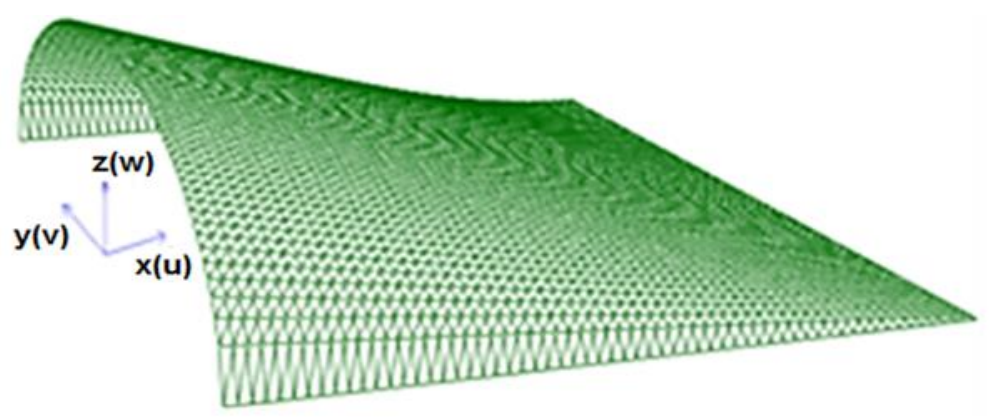

Figura 4. Malha de elementos finitos STRI 3 para Geometria 1.

\section{Análise estática não linear dos esforços de membrana}

Uma análise essencial para o dimensionamento de estruturas de coberturas é a variação dos esforços solicitantes internos. Os esforços de membrana geram compressão, ou tração, e cisalhamento em pontos diferentes da superfície. Nesta seção, avalia-se a variação da trajetória de equilíbrio dos esforços normais e de cisalhamento ao longo da incrementação de carregamento, comparando-se as geometrias 1 e 2 . Percebe-se que a variação das alturas das bordas produzem trajetórias não lineares diferenciadas. Para este estudo utilizou-se o ponto de observação localizado no centro dos conóides, além dos pontos de observação na trajetória de equilíbrio denominados $\mathrm{M}_{1}$, $\mathrm{M}_{2}$ e $\mathrm{M}_{3}$ posicionados de forma a ilustrar os esforços na mesma coordenada de carregamento para as Geometrias consideradas.

Através da figura 5, podem-se verificar como as não linearidades geométricas das equações de equilíbrio influenciaram para a obtenção de trajetórias não lineares entre as geometrias para os esforços normais, $N_{x}$. Nota-se que, em pontos inferiores a $200 \mathrm{kN} / \mathrm{m}^{2}$ de carga, $\mathrm{M}_{1}$, a Geometria 1 apresenta este esforço com magnitudes superiores à Geometria 2.

A Geometria 1 é caracterizada pelas regiões das bordas "a" serem predominantemente comprimidas e pela existência de regiões com picos de tração nas proximidades das bordas curvas $\mathrm{H}_{2}$. Nota-se, em $\mathrm{M}_{1}$ na Geometria 2, a existência de tração no centro das bordas extremas com compressão nos quatro cantos no início da trajetória, cenário este que se modifica ao incrementar carregamento, $\mathrm{M}_{2}$ e $\mathrm{M}_{3}$, cuja compressão passa a ser presente em faixas paralelas próximas às bordas retas e a tração preponderante no centro da casca. Observa-se ainda que o esforço de membrana $\mathrm{N}_{\mathrm{x}}$ é simétrico em torno do eixo $\mathrm{x}$ e em torno do eixo y é simétrico para a Geometria 2 e assimétrico para a Geometria 1. 


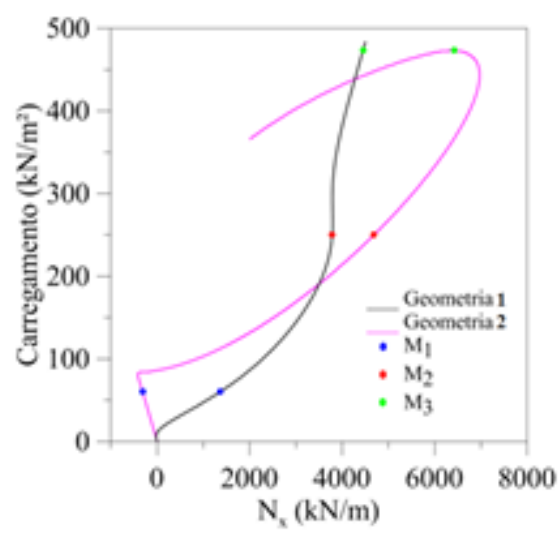

Geometria 1

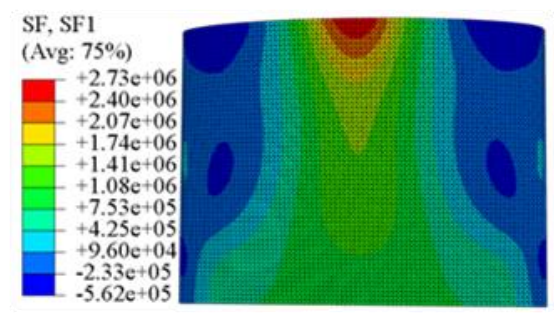

(b) $\mathrm{M}_{2}$

(c) $\mathrm{M}_{3}$


Geometria 2
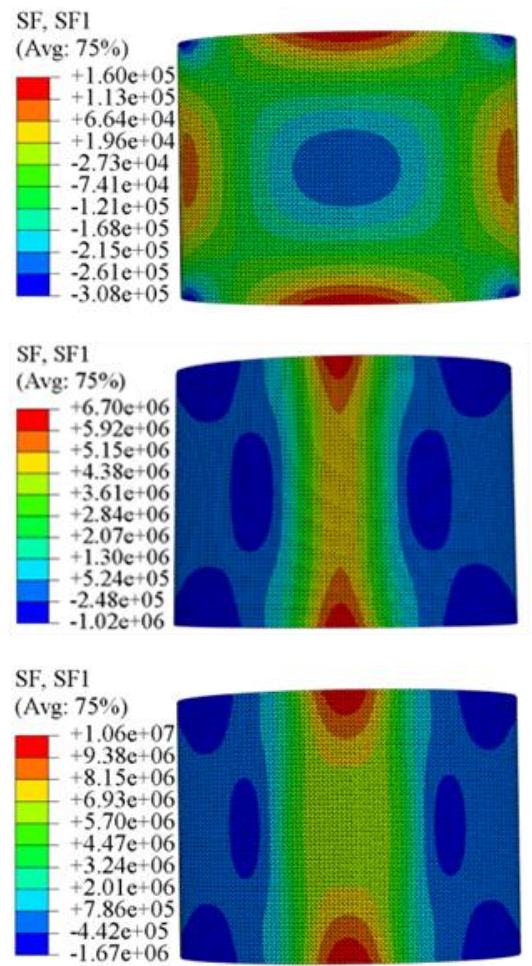

Figura 5. Trajetória não linear de $\mathrm{N}_{\mathrm{x}}$ do conóide cilíndrico

conóides são os esforços normais $N_{y}$ ilustrados na figura 6.

lineares de grandes magnitudes em várias regiões dos 


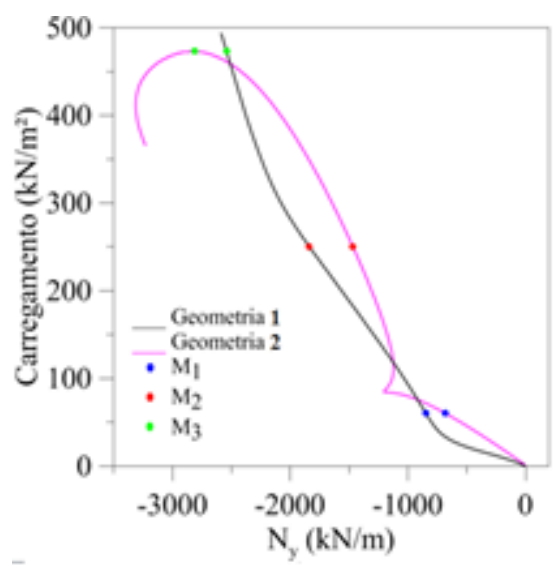

Geometria 1

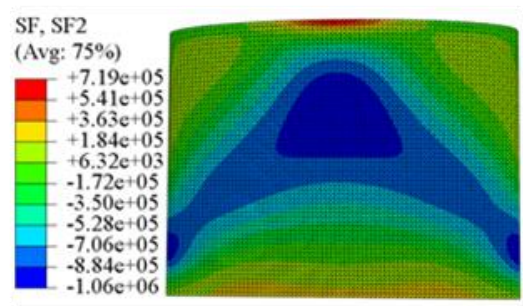

(b) $\mathrm{M}_{2}$

(c) $\mathrm{M}_{3}$

(a) $\mathrm{M}_{1}$

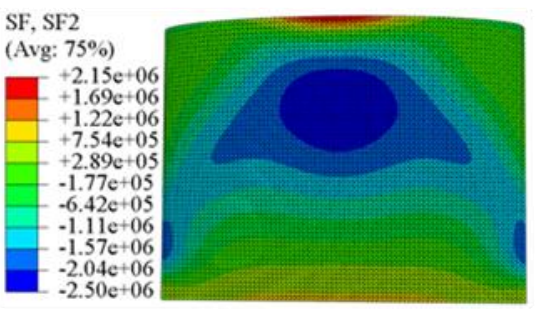

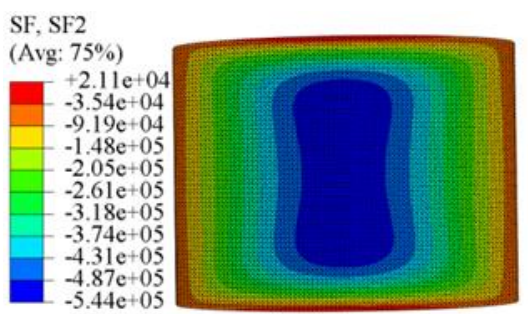
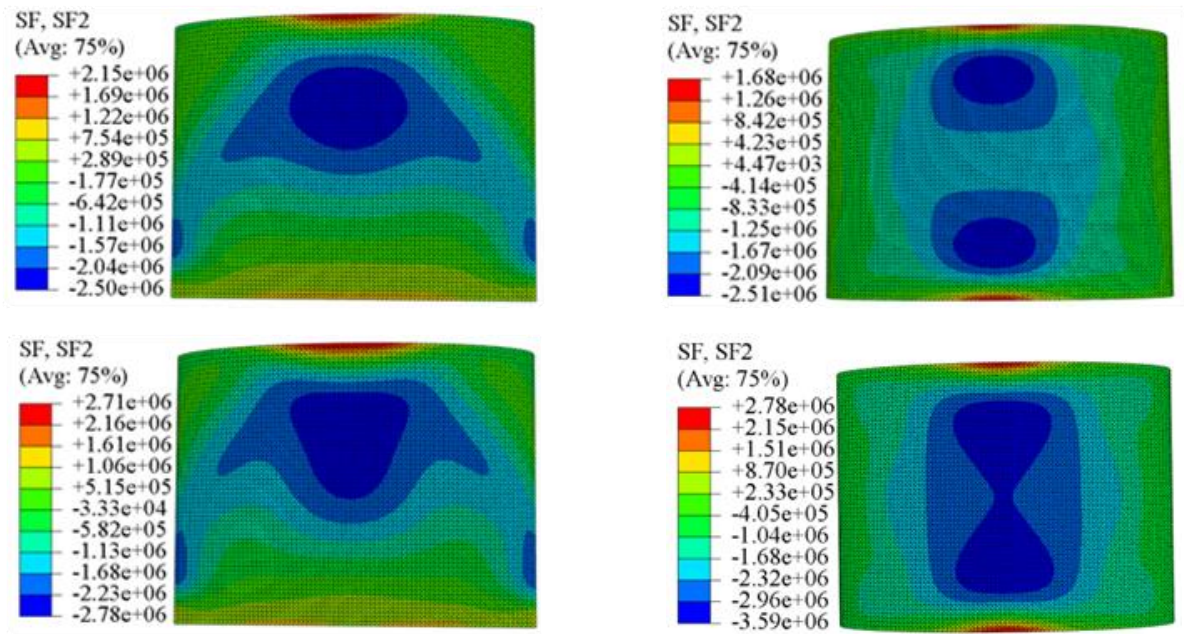

Figura 6. Trajetória não linear de $\mathrm{N}_{\mathrm{y}}$ do conóide cilíndrico

Percebe-se a distribuição simétrica de $N_{y}$ em torno do eixo y e, na Geometria 1, a existência de faixas de compressão não lineares entre as bordas "a", além de tração, de menor concentração, nas bordas $\mathrm{H}_{\mathrm{n}}$. Já no conóide de Geometria 2, observou-se uma alta concentração de compressão na parte central durante toda a trajetória de equilíbrio.

$\mathrm{Na}$ Figura 7, podem ser visualizadas as distribuições dos esforços normais cisalhantes no plano, $N_{x y}$. Verifica-se que, no ponto estudado, as magnitudes do esforço cisalhante são inferiores aos demais esforços de membrana havendo regiões de picos próximos às bordas curvas. Observa-se ainda, como esperado, a distribuição antissimétrica em torno do eixo y e em torno do eixo $x$ : antissimetria na Geometria 2 e assimetria na Geometria 1. 


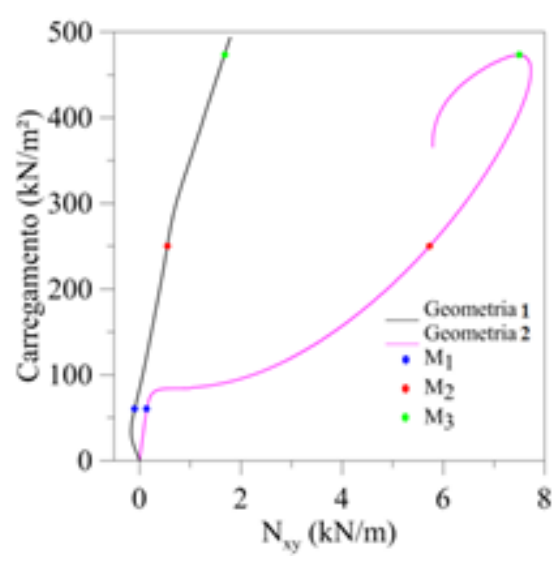

Geometria 1

Geometria 2

(a) $\mathrm{M}_{1}$
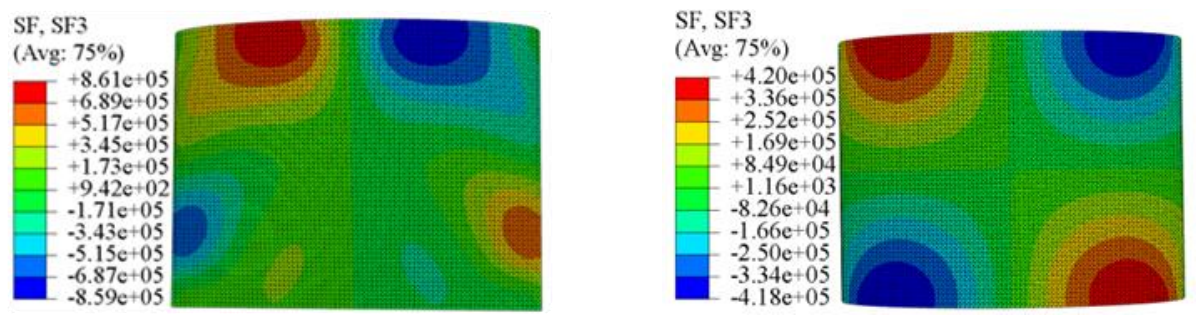

(b) $\mathrm{M}_{2}$
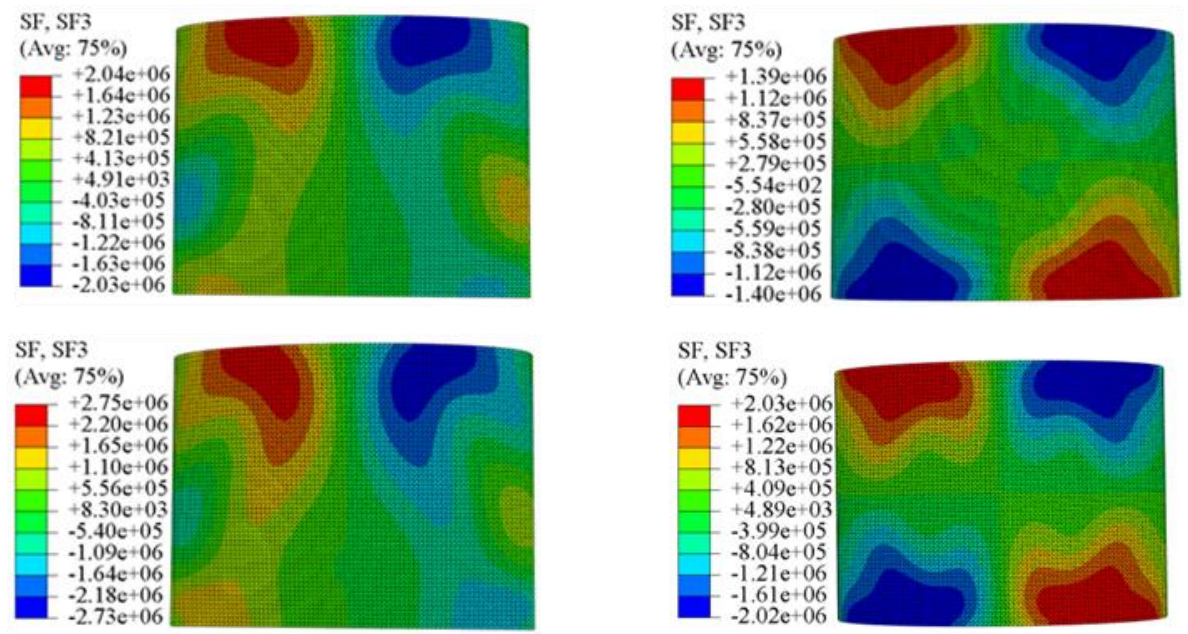

Figura 7. Trajetória não linear de $\mathrm{N}_{\mathrm{xy}}$ do conóide cilíndrico

Por fim, destaca-se que no ponto de observação, $\mathrm{P}_{3}$, em todos os conóides a incrementação do carregamento transversal provocou maiores valores das resultantes de membrana no ponto de observação na trajetória de equilíbrio $\mathrm{M}_{1}$. A seguir, destacam-se as considerações observadas a cerca da trajetória não linear dos momentos internos.

\section{Análise estática não linear nos momentos internos}

Outra análise crucial para o dimensionamento de estruturas de coberturas é a variação dos momentos internos. Nesta seção verificam-se como as não linearidades geométricas das equações de equilíbrio influenciaram para a obtenção de comportamento não linear ao longo da incrementação de carga. $\mathrm{Na}$ figura 8 observam-se as diferenciadas trajetórias dos conóides quando a altura da borda $\mathrm{H}_{1}$ é alterada, verifica-se no ponto de análise $\mathrm{M}_{1}$ que a Geometria 1 conferiu 
maiores esforços solicitantes em relação a Geometria 2.

No entanto, no decorrer da incrementação de carga este

cenário varia não linearmente.

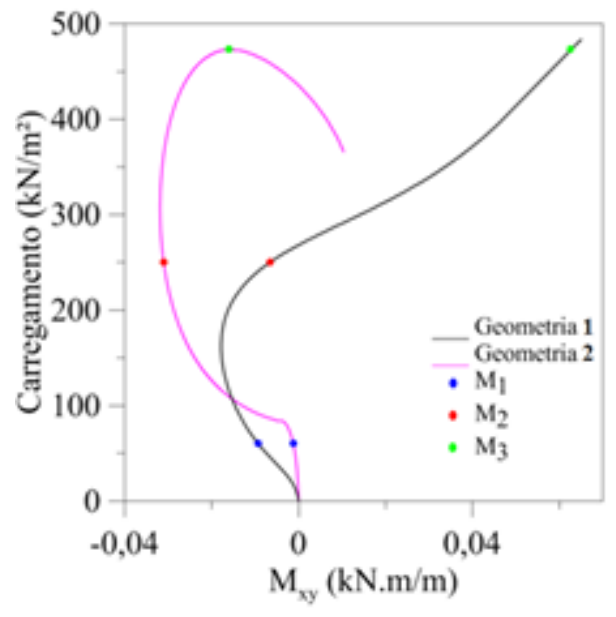

(a)

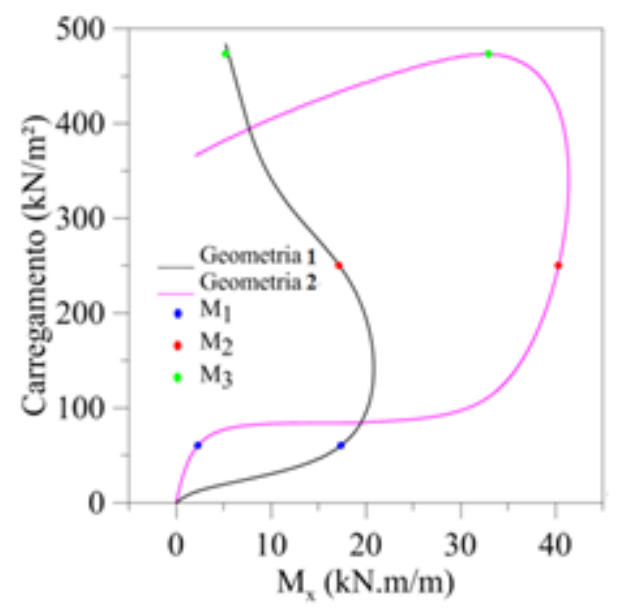

(b)

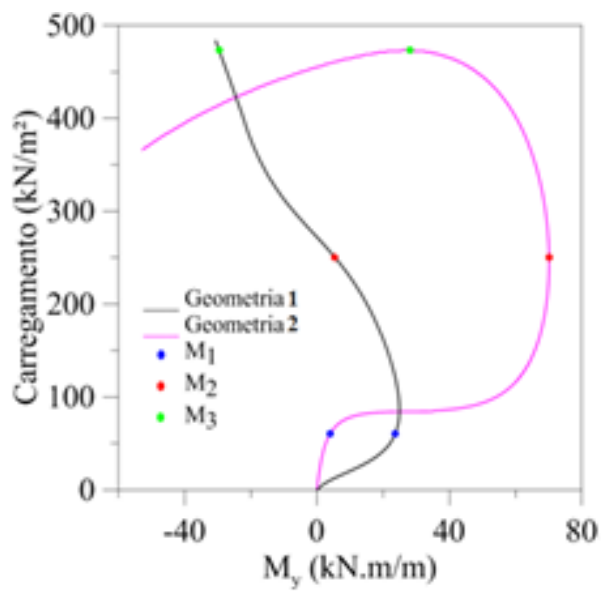

(c)

Figura 8. Trajetória não linear dos esforços solicitantes (a) Torção (b) Flexão em x (c) Flexão em y.

$\mathrm{Na}$ Figura 9 são apresentadas as topologias das trajetórias não lineares do momento torçor, $M_{x y}$. Observa-se a distribuição praticamente uniforme na parte central das cascas conoidais e picos máximos próximos às bordas curvas. É interessante destacar o comportamento das topologias, nas quais os momentos torçores se distribuem de forma antissimétrica, em torno dos eixos $x$ e $y$, e também nas quais o incremento de carregamento provocou nos conóides de Geometria 1 maiores distribuições dos picos máximos ruma a superfície central do conóide devido à inclinação entre as borda curvas. 


\section{Geometria 1}

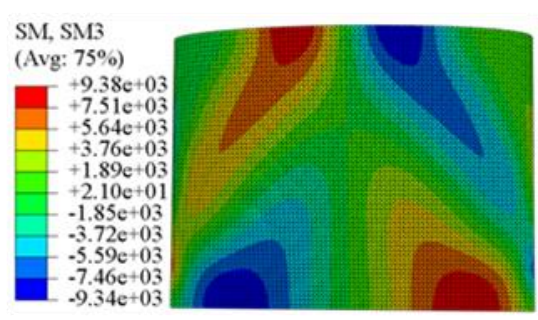

(b) $\mathrm{M}_{2}$

(a) $\mathrm{M}_{1}$

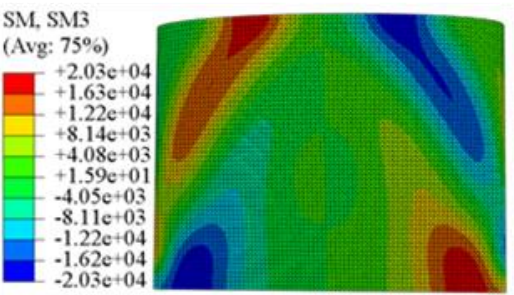

(c) $\mathrm{M}_{3}$

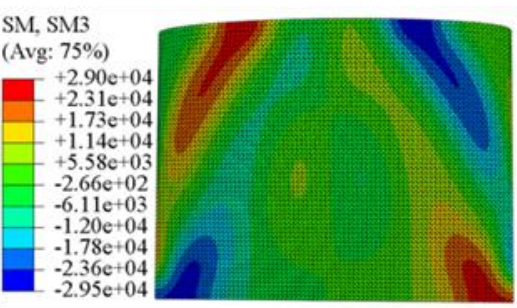

Geometria 2
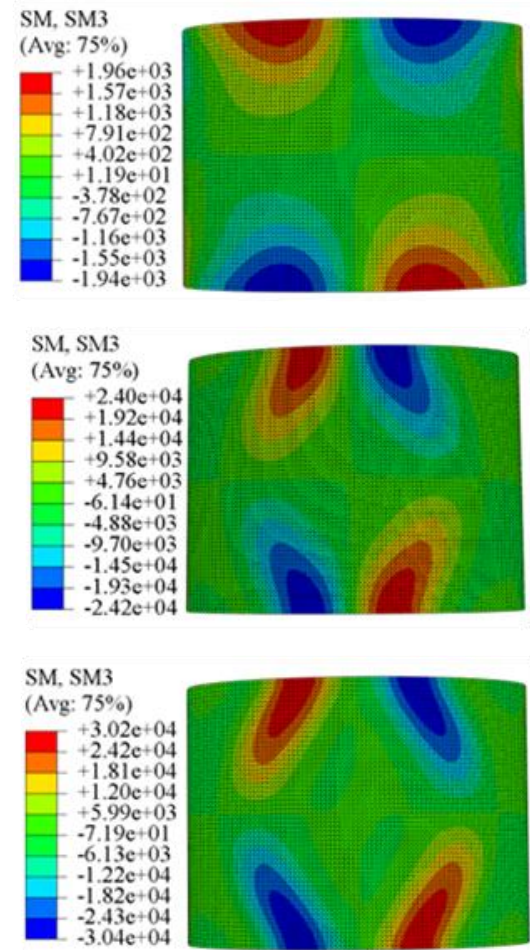

Figura 9. Trajetória não linear de $\mathrm{M}_{\mathrm{xy}}$ do conóide cilíndrico

O comportamento não linear das topologias do momento fletor, $M_{x}$, pode ser visualizado na figura 10 e do momento $M_{y}$, na figura 11 . Visualizam-se nestas duas figuras que a distribuição destes esforços é simétrica, em torno do eixo $y$, independentemente da geometria. Nota-se ainda que na Geometria 1 há uma distribuição maior de momentos positivos na região central em relação à Geometria 2, porém nas duas geometria visualiza-se uma concentração de momentos negativos próximos às bordas retas "a" e momento positivos na parte central. É interessante ressaltar que ao longo da trajetória de equilíbrio, o número de semiondas aumenta, o que sugere a influência de diferentes modos de vibração para manter o equilíbrio com o aumento de carga. 
Geometria 1

(a) $\mathrm{M}_{1}$
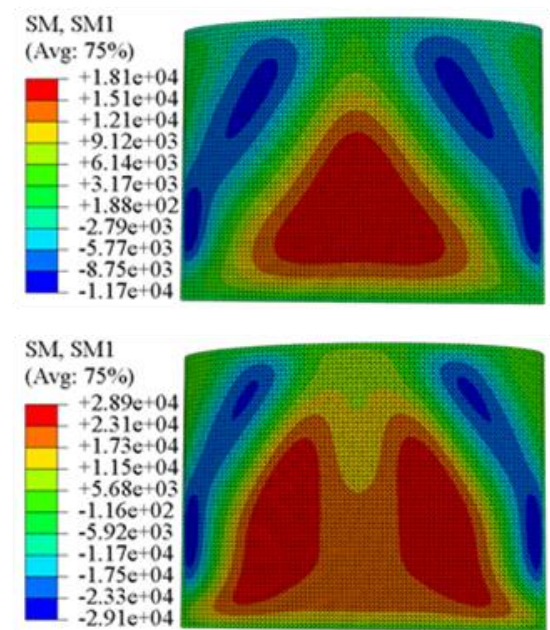

(b) $\mathrm{M}_{2}$

(c) $\mathrm{M}_{3}$

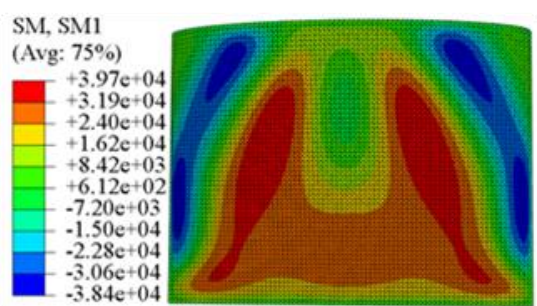

\section{Geometria 2}
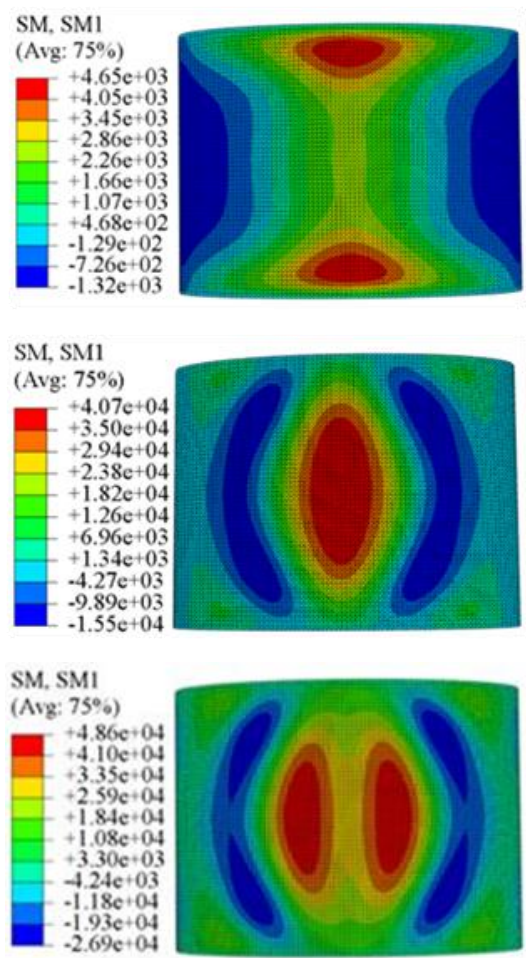

Figura 10.- Trajetória não linear de $\mathrm{M}_{\mathrm{x}}$ do conóide cilíndrico

Geometria 1

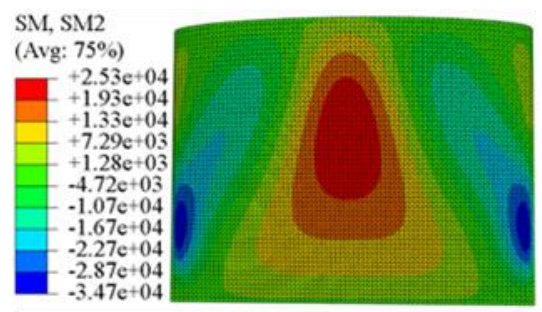

(b) $\mathrm{M}_{2}$

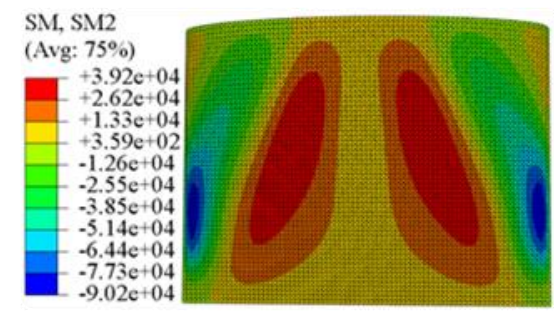

(c) $\mathrm{M}_{3}$

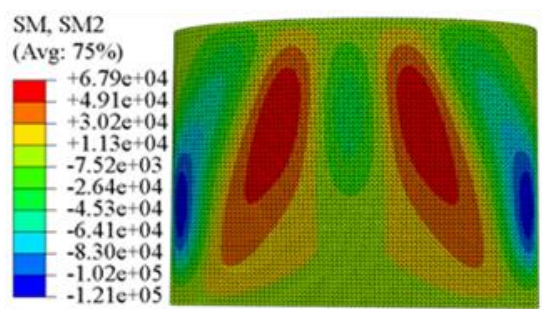

Geometria 2
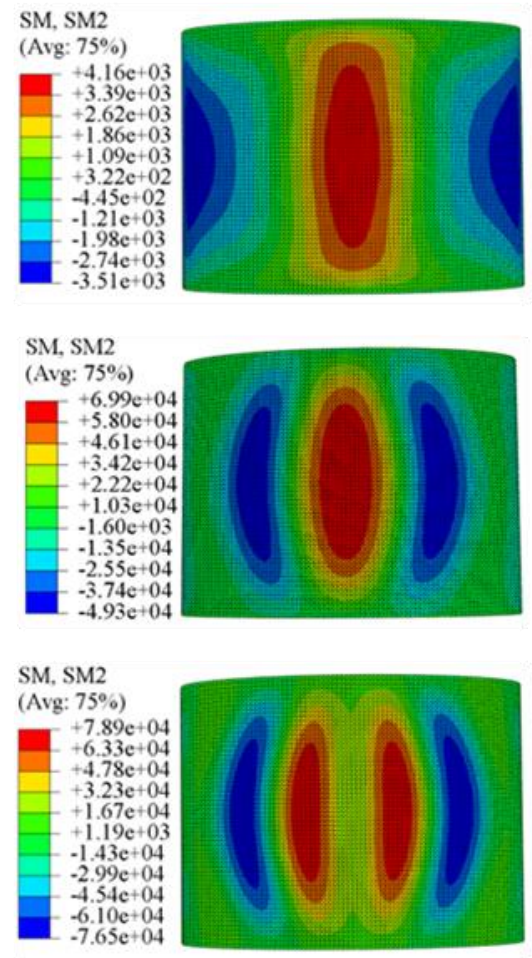

Figura 11. Trajetória não-linear de $\mathrm{M}_{\mathrm{y}}$ do conóide cilíndrico 


\section{CONCLUSÕES}

A utilização do MEF tornou possível a solução aproximada do problema de difícil solução analítica do conóide cilíndrico apresentada neste trabalho. Este estudo evidencia a importância da consideração das não linearidades geométricas. Em conjunto com a variação das alturas das bordas curvas do conóide resulta em comportamentos altamente não lineares. Nos quais as posições de pico dos esforços solicitantes, importantes ao dimensionamento estrutural, variam ao longo da incrementação de carga.

\section{REFERÊNCIAS}

ABAQUS 6.11. ABAQUS/CAE User's Manual. Dassault Systemes, USA, 2011.

Bandyopadhyay, J. N.; Chakravorty, D.; Sinha, P. K. 1995, Finite element free vibration analysis of conoidal shells, Computers \& Structures, v. 56, n. 6, p. 975-978.

Chakravorty, D.; Das, H. S. 2009, A finite element application in the analysis and design of pointsupported composite conoidal shell roofs: suggesting selection guidelines, Departamento de Engenharia Civil, Univesidade de Jadavpur, Kolkata, Índia.

Morais, D. L. A. 2017, Análise estática não-linear de cascas conoidais, Dissertação de Mestrado, PPG-GECON/UFG, Goiânia.

Sahoo, S. 2013, Dynamic characters of stiffened composite conoidal shell roofs with cutouts: design aids and selection guidelines, Hindawi
Publishing Corporation Journal of Engineering, p. 18. ID 230120

Cavalcanti, L. V. 2015, Análise da estabilidade e vibrações de cascas conoidais abatidas, Dissertação de Mestrado em Engenharia Civil, Pontifícia Universidade Católica do Rio de Janeiro, Rio de Janeiro, p. 154.

Gonçalves, P. B.; Soares, R. M (2016), Análise numérica do comportamento não-linear estático e dinâmico de cascas conoidais, In: Proceedings of the 37th Cilamce Iberian Latin-American Congress on Computacional Methods in Engineering, Brasília.

Bandyopadhyay, J. N. A. Ghosh, B. 1990, Approximate bending analysis of conoidal shells using the Galerkin method, Computers e Structures, Great Britain, 36, n. 5, p. 801-805.

Stavridis, L. T. 1998, Dynamic analysis of shallow shells of rectangular base, Journal of Sound an Vibration, 861-882, sv981907.

Debongnie, J. F. 1978, Physical interpretation and generalization of Marguerre's shallow shell theory, Int J. Eng Sci. v. 17, p. 387-399.

Almroth, B. O.; Brush, D. O. 1975, Buckling of Bars, Plates and Shells. USA: McGraw-Hill Book Company, Inc.

STRUCTURAE. Felix Candela: Biographical Information. International Database for Civil and Structural Engineering. Disponível em: $<$ https://structurae.net/persons/felix-candela> Acesso: 25/03/2016

FGV. Torre Oscar Niemeyer: Galeria de fotos. Fundação Getúlio Vargas. Diponível em: $<$ http://torre-oscar-niemeyer.fg v.br/galeria> . Acesso: 25/03/2016. 